\title{
Apparent hyperglycemic and hypoglycemic conditioned responses with exogenous insulin as the unconditioned stimulus
}

\author{
CHARLES F. FLAHERTY, ANTHONY J. UZWIAK, JAMI LEVINE, \\ MARYANNE SMITH, PAMELA HALL, and ROBYN SCHULER \\ Rutgers University, New Brunswick, New Jersey 08903
}

\begin{abstract}
Rats were injected with either insulin or saline and placed in one of two distinctive environments for $25 \mathrm{~min}$ on each of several days. On test trials for conditioning, the animals were injected with saline in both environments and blood glucose levels were measured. Apparent conditioned changes were obtained, but the direction of the conditioned response depended upon the environment in which the animals were placed. A conditioned hyperglycemic response occurred in one environment, and a conditioned hypoglycemic response occurred in the other environment. This pattern occurred in both within-subject and between-subjects designs and was not influenced by route of insulin injection (ip or sc). The hypoglycemic, but not the hyperglycemic, response was blocked by methyl atropine. The occurrence of both CR patterns with the same low insulin dose is contrary to previous speculation that the different CR patterns are due to different dose levels of insulin.
\end{abstract}

It has been shown that repeated injection of insulin in a constant environment will lead to the development of a conditioned response (CR) that is elicited by the environment itself (Alvarez-Buylla and De Alvarez-Buylla, 1961; Siegel, 1972, 1975a; Woods, 1972, 1976). The general procedure used in these experiments was as follows. The subjects (rats or dogs) were injected with insulin (doses ranged from .05 to $50 \mathrm{U} / \mathrm{kg}$ in different experiments) and placed in a particular stimulus context for approximately $20 \mathrm{~min}$. After several injections (usually every other day for 4-6 days), the animals were injected with physiological saline and placed in the same stimulus context. At the end of the 20 -min period, a blood sample was taken. Control conditions included animals injected with saline throughout the experiment and blood samples taken before and after the injections in both control and experimental animals.

The results of these experiments have shown that the insulin injection itself lowers blood glucose concentration (as would be expected). Many studies have also shown that there is a drop in blood glucose concentration when saline is injected into animals with a history of insulin injections in a particular environment. That is, the environment acts as a conditioned stimulus (CS) that elicits a CR similar in form to the unconditioned response (UCR) produced

This work was supported by Grant R03MH32032 from the National Institute of Mental Health and by a grant from the Charles and Johanna Busch Bequest to Rutgers University. We would like to thank Drs. Lung-Hsiung $\mathrm{Hsu}$ and Mei Hsu of Worthington Biochemicals for their assistance and suggestions concerning blood glucose measurement. by the insulin injection (e.g., Alvarez-Buylla \& De Alvarez-Buylla, 1975; Woods, 1972, 1976; Woods, Makous, \& Hutton, 1968, 1969).

However, a series of studies by Siegel $(1972,1975 \mathrm{a})$, using essentially the same procedures as those of the above studies, obtained evidence for a CR that was in the opposite direction from the UCR. That is, when animals with a history of insulin injection in a particular environment were injected with saline in that environment, there was a rise in blood glucose levels above those seen in control animals. Evidence for such CRs that take the form of apparently anticipatory compensatory changes have been obtained in conditioning studies involving the intubation of glucose (Deutsch, 1974), the administration of opioid drugs (Eikelboom \& Stewart, 1979; Krank, Hinson, \& Siegel, 1979; Siegel, 1975b, 1976, 1977, 1978; Siegel, Hinson, \& Krank, 1979), and alcohol (Hinson, Crowell, \& Siegel, 1979) as well as in more traditional Pavlovian procedures (e.g., Fitzgerald, Martin, \& O'Brien, 1973).

Thus, in the case of insulin injections, there exist two data sets, each comprising a substantial number of studies, finding diametrically opposite results in regard to the nature of the CR obtained. One set of results, those showing conditioned hyperglycemia, is consistent with the hypothesis that the $C R$ functions as an anticipatory compensatory response serving to offset deviations from steady state produced by the UCS (cf. Siegel, 1977). However, those studies finding a conditioned hypoglycemic response are not consistent with this hypothesis

In the present paper, we report the results of three experiments investigating glucoregulatory condition- 
ing using exogenous insulin as the UCS. In these studies, we obtained both sets of results described above, that is, conditioned hyperglycemia and conditioned hypoglycemia, each correlated with a different stimulus complex.

\section{EXPERIMENT 1}

In this experiment we adopted a within-subject conditioning paradigm. That is, all animals were injected with both insulin and saline, but each type of injection was associated with a different stimulus context. The different injections were given on alternate days, with a day off after each 2 injection days. After a series of conditioning trials, the animals were tested for the presence of a CR by the administration of saline in both the environment normally paired with saline and the environment normally paired with insulin.

\section{Method}

Animals. Twenty naive, male, Sprague-Dawley-derived rats, purchased from Charles River Breeding Farms, were used as subjets. The rats were individually housed and were maintained on a 14/10-h light/dark cycle. Food and water were continuously available. The rats weighed between 373 and $440 \mathrm{~g}$ at the start of the experiment.

Apparatus. Two different conditioning environments were used. These different environments, described in Table 1, were set up in two different rooms of the laboratory. The wastebaskets used in Environment A were black plastic, and each had a piece of wood paneling laid across the top as a cover. The metal cages used in Environment B were standard rat-housing cages (Wahmann) and were placed over newspapers on a table top. These cages also each had a piece of wood paneling as a cover.

Procedure. On each conditioning day, the animals were weighed, brought from their home cages to one of the environments, given the appropriate injection for that environment, confined to the apparatus for $25 \mathrm{~min}$, and then returned to their home cages. This procedure was carried out in a series of 3-day cycles. The Ist day of a cycle was either an insulin or saline injection (determined randomly); on the 2 nd day, the alternative injection was given; the 3rd day was an off-day. All injections were subcutaneous (sc) in Environment $A$ and intraperitoneal (ip) in Environment B. The animals were given eight of these conditioning cycles. For half of the animals, insulin was injected, in Environment $\mathbf{A}$, and for the remaining animals, insulin was injected in Environment B. Saline was always administered in the alternative environment. The insulin was a $2.7 \mathrm{U} / \mathrm{kg}$ dose of regular insulin purchased from Sigma Chemicals. The subcutaneous injection was given in the back of the neck.

Test trials for conditioning were given following the acquisition series. On these trials, the animals were injected with saline in both environments. Two days were required for these tests and, over these 2 days, environmental condition was counterbalanced with order of testing. That is, half of the animals that received insulin in Environment $A$ were given a test trial in that environment on the 1st test day, and the remaining animals were given their first test trials in Environment $\mathrm{B}$-their saline environment. Similar counterbalancing procedures were used for the animals that received insulin in Environment B.

Blood glucose sampling and measurement procedures were as follows: Upon removal from the conditioning environment on test days, the rats were returned to the colony room and placed on a table, where they were gently restrained by one experimenter. A second individual removed the tip of the rat's tail with a scalpel and withdrew $75 \mu \mathrm{l}$ of blood into a heparin-coated microhematocrit tube. The sampling procedure usually required from 15 to $45 \mathrm{sec}$.

The samples were centrifuged and blood glucose levels were determined by the coupled enzyme procedure (Bondar \& Mead, 1974). For this purpose, we used the Satzyme Glucose reagent kit purchased from Worthington Diagnostics (Freehold, New Jersey).

\section{Results}

The mean blood glucose levels (BGL) obtained on test days as a function of drug and environmental conditions are shown in Figure 1. The data indicate that the nature of the CR obtained when saline was administered in an environment previously paired with insulin varied as a function of the characteristics of the environment. The animals that had received insulin in Environment A and saline in Environment B showed a hyperglycemic $C R$, whereas the rats that had received insulin in Environment $B$ and saline in Environment A showed a hypoglycemic CR. The reliability of this pattern of results was confirmed in a two-way analysis of variance. The analysis indicated a reliable interaction between drug condition and environment $[F(1,15)=10.09, p<.01]$. Subsequent analysis of this interaction using Fisher's least significant difference (l.s.d.) test (Li, 1964) indicated the following pattern of reliability. The within-subjects comparison of the animals that had received insulin in Environment $\mathrm{A}$ showed a reliable conditioned hyperglycemic effect (test in A > test in B), and the within-subjects comparison of the animals that had received insulin in Environment $B$ showed a reliable conditioned hypoglycemic effect (test in B $<$ test in A).

In addition, the between-subjects comparison of the animals that had received insulin in $B$ vs. those that had received saline in $B$ also indicated a reliable

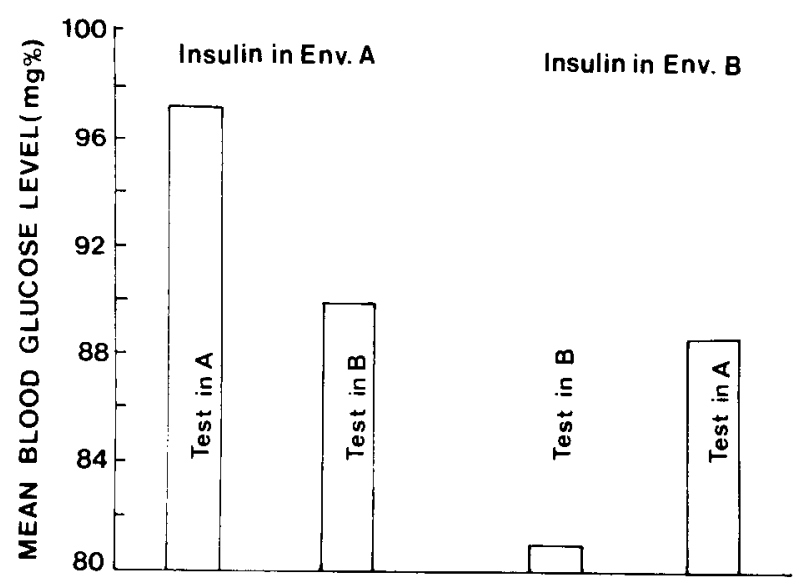

Figure 1. Mean blood glucose levets obtained on test days, when all animals were injected with saline. The lefimost bar presents the data obtained when animals that usually received insulin in Environment $A$ were given saline in that environment. The next bar shows the data for these same animals when given saline in their ustual saline environment (B). The last two bars depict the data for animals usually given insulin in Environment $B$. 
hypoglycemic effect by the LSD test $(p<.05)$. Finally, the between-subjects comparison of the animals that had received insulin in $A$ vs. the animals that had received saline in $A$ was marginally reliable by $L S D$ test $(p<.07)$.

It is apparent from Figure 1 that the BGLs of the animals receiving saline in the two enviroments were not different.

\section{Discussion}

The previous literature had indicated contradictory results in regard to the nature of the $C R$ obtained when insulin is used as the UCS, many studies having found a hypoglycemic CR, but others having found a hyperglycemic $C R$, using essentially the same procedures. In the present study, two environments were established to differ in a number of dimensions so as to establish the basis of rapid differential conditioning. The occurrence of apparent hyperglycemic conditioning in one environment and hypoglycemic conditioning in the other was not anticipated, particularly in light of the recent argument by Woods and Kulkosky (1976) that the hypoglycemic CR may be found only with large (25-50 U/ kg) doses of exogenous insulin, whereas the hyperglycemic CR may be found only with small doses. In the present study, we obtained both patterns with a "small" dose.

In order to obtain some information as to whether the hypoglycemic CR found in our experiment might be functionally similar to that obtained by Woods and his colleagues, we replicated a procedure of theirs.

\section{EXPERIMENT 1A}

Woods (1972) reported that cervical vagotomy or injections of atropine methyl bromide served to eliminate a conditioned hypoglycemic response. The interpretation of this result was that the CR was normally mediated by the endogenous release of insulin under the influence of cholinergic fibers of the vagus (Chen, Woods, \& Porte, 1975; Woods, 1972; Woods \& Porte, 1974, 1975). When this release is prevented from occurring, the CR is blocked.

In the present experiment, we examined the effects of atropine injections on the hyper- and hypoglycemic CRs obtained in Experiment 1.

\section{Method}

The animals and apparatus were the same as in Experiment 1.

Following a 1-week break in training, six additional conditioning trials were administered to the animals used in Experiment 1. Three of these trials were in each environment, following exactly the same procedure as used in Experiment 1. Test trials for conditioning were conducted as in Experiment 1 , except that half of the animals in each group were administered $5 \mathrm{mg} / \mathrm{kg}$ of atropine (atropine methyl nitrate, Sigma Chemicals) instead of saline as the "placebo" injection.

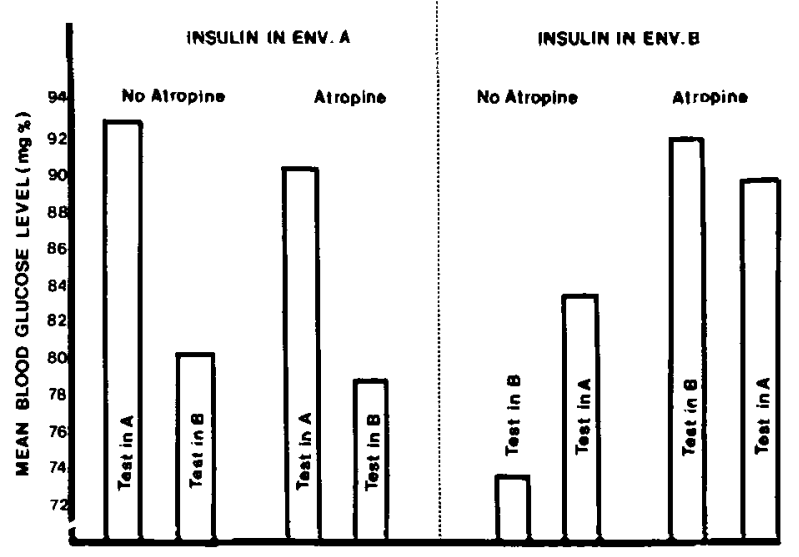

Figure 2. Mean blood glucose levels obtained on lest days, when the rats were injected with either saline or atropine. Labeling conventions are as in Figure 1.

\section{Results}

Two animals were dropped from the study, one because of difficulty in injecting and one because of difficulty during the blood sampling procedure. Since the results of interest were known from Experiment 1 and from the Woods (1972) study, an individual degrees of freedom analysis of variance was conducted (Li, 1964).

The results obtained on the test day are presented in Figure 2. It is clear that a hyperglycemic CR was again obtained in Environment $\mathbf{A}$, both under condi-; tions of no atropine $[F(1,14)=8.95, p<.01]$ and under the atropine injection $[\mathrm{F}(1,14)=11.36, \mathrm{p}<.01]$. In Environment $\mathrm{B}$, a hypoglycemic $\mathrm{CR}$ was also obtained, again under the no-atropine condition $[F(1,14)$ $=6.64, p<.05]$, but injections of atropine eliminated the hypoglycemic $C R(F<1)$.

\section{Discussion}

The similarity of the effect of atropine on the hypoglycemic $C R$ in this experiment and in the Woods (1972) study indicated that the hypoglycemia found with the low dose of insulin and in the withinsubjects conditioning procedure is functionally similar to that obtained by Woods with the high insulin dose and in the between-subjects procedure. The fact that atropine had no effect on the hyperglycemic CR would indicate that a noncholinergic mechanism is involved in this response.

Finally, the occurrence of both a hyper- and hypoglycemic $C R$ once again with the same insulin dose indicates that the suggestion of Woods and Kulkowsky (1976), that the different CR patterns are dose related, is no longer tenable.

\section{EXPERIMENT 2}

In the present experiment, we were concerned with (a) whether the opportunity for the rats to experience 
Table 1

Characteristics of the Two Environments Used as Conditioned Stimuli

\begin{tabular}{ll}
\multicolumn{1}{c}{ Environment A } & \multicolumn{1}{c}{ Environment B } \\
\hline Wastebasket .36 $\mathrm{m}^{3}$ & Metal Grid Cage .09 $\mathrm{m}^{3}$ \\
Wood Chips & No Wood Chips \\
Menthol Odor & No Menthol Odor \\
Dim Incandescent Lights & Bright Fluorescent Lights \\
White Noise & No White Noise \\
Subcutaneous Injection & Intraperitoneal Injection \\
Dark Wooden Carrying Case & Light Wooden Carrying Case \\
\hline
\end{tabular}

Note-The wastebasket and metal cage served as holding devices for the 25-min confinement period in each environment.

both environments was necessary for the hyper- and hypoglycemic patterns to develop, and (b) whether the difference in $C R$ pattern obtained in the two environments might be due to the route of insulin injection.

In examining the differences between the two environments indicated in Table 1 the difference in route of injection seemed most likely to be responsible for the different patterns of CR occurring in A and B. Route of injection was particularly salient because of possible differences in time required for absorption of the insulin and because of possible differences in stress associated with the injection procedure, the sc procedure seeming to produce more disturbance in the rats. In order to obtain some information concerning this possibility, all injections in the present experiment were made ip.

\section{Method}

Animals. The suojects were 48 male Sprague-Dawley-derived rats maintained on ad-lib food and water.

Apparatus. The apparatus was the same as that used in the previous experiments.

Procedure. The rats were assigned randomly to the following four groups (12 per group): insulin in A, saline in A, insulin in B, saline in $B$. Seven acquisition trials were administered, one on each of 7 consecutive days. On each day, the animals were carried into the appropriate environment, given the appropriate injection (ip in both environments), placed in the holding structures (cages or wastebaskets) for $25 \mathrm{~min}$, and then removed and returned to the colony room. In the colony room, each rat was allowed to roam freely on a paper-covered table top for approximately $30 \mathrm{sec}$ before being returned to the home cage. The insulin injections were 2.68 I.U. of regular insulin purchased from Sigma Chemicals. New insulin was prepared, by mixing with physiological saline, every other day. The insulin was refrigerated when not in use.

On the day following the 7th acquisition day, the animals were given a test trial for conditioning. On this day, the procedure was the same as above, except that all animals were injected with saline. When the animals were removed to the colony room on the test day, the procedure was the same as above, except that when the animals were placed on the table top they were gently restrained while a second experimenter withdrew a blood sample, as in the earlier experiments. Blood glucose analysis was also conducted as in the earlier experiments.

\section{Results and Discussion}

Two animals were dropped from the experiment because of difficulty with the injection procedure.
The mean blood glucose levels obtained on the test day are presented in Figure 3. It is readily apparent that the same pattern of hyperglycemia in A and hypoglycemia in B obtained in the earlier experiments occurred with the between-subjects procedure used in the present study. An analysis of variance performed on the data shown in Figure 3 indicated a reliable Drug by Environment interaction $[F(1,38)=$ $13.19, p<.001]$. Subsequent analysis of this interaction using the Fisher l.s.d. procedure indicated that both the hyperglycemic CR pattern in A ("insulin" > saline) and the hypoglycemic CR pattern in B ("insulin" $<$ saline) were statistically reliable $(p<.05)$. The blood glucose levels of the animals injected with saline in the two environments were not reliably different.

The results of the present experiment confirm the findings of the previous two experiments and show that the pattern of a hyperglycemic CR in Environment $\mathrm{A}$ and a hypoglycemic CR in Environment B is not unique to a within-subjects administration used in the two environments in the previous experiments.

\section{GENERAL DISCUSSION}

As reviewed in our introductory comments, the previous literature had indicated two distinctly different patterns of CR obtained with insulin injection as the UCS. One set of investigators consistently obtained a hyperglycemic $C R$, even when attempting to replicate conditions under which the hypoglycemic pattern was obtained by others. In the series of experiments presented above, we have been able to replicate both

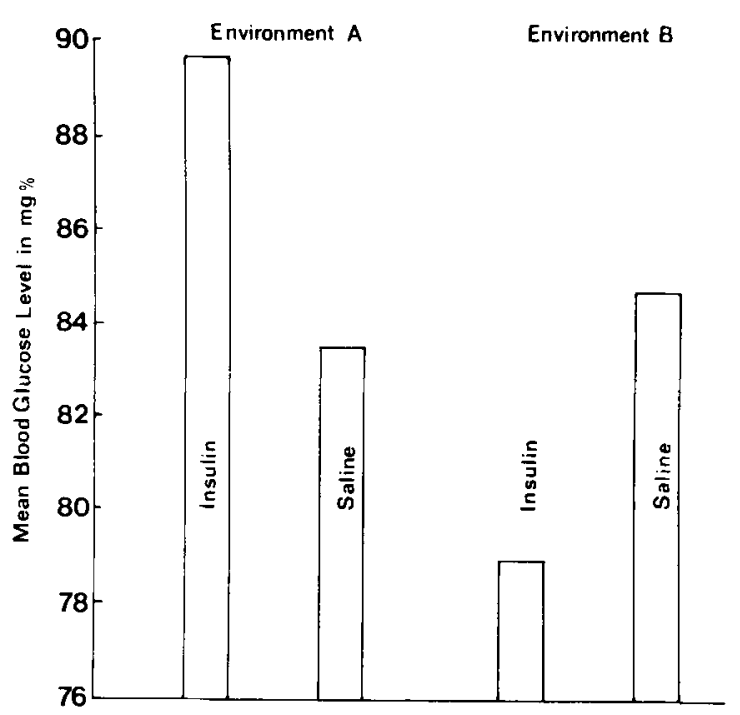

Figure 3. Mean blood glucose levels obtained on the lest day. The two hars on the left present data for animals always placed in Environment $A$. The lefimost of these bars presents the data obtained when animals that had been given insulin in finvironment $A$ were given saline. The next bar presents the data for animals that had alway received saline in $A$. The last two hars present the data for animals given insulin or saline in Environment $B$. 
aspects of these contradictory results and have shown that the occurrence of both patterns in the same experiment is not uniquely restricted to within-subjects designs and it not due to differences in route of drug injection. Furthermore, the results of these experiments indicate that a hypoglycemic $\mathrm{CR}$ is not restricted to high doses of insulin and that the hypoglycemic CR obtained with the low insulin dose used in the present experiments may be functionally similar to that obtained with high insulin doses in the previous literature.

The occurrence of both hyperglycemic and hypoglycemic CRs under the conditions of the three experiments reported here indicates that some subtle environmental condition may interact with the effects of the insulin injection in producing one or the other pattern of response. The reliable occurrence of both $C R$ patterns in the present paradigm should permit the isolation of this environmental condition as well as the investigations of the physiological mechanisms responsible for the two CR patterns.

\section{REFERENCE NOTES}

1. Hinson, R. E., Crowell, C., \& Siegel, S. Pavlovian conditioning mediates tolerance to ethanol-induced hypothermia. Paper presented at the meeting of the Eastern Psychological Association, Philadelphia, 1979.

2. Krank, M. D., Hinson, R. E., \& Siegel, S. Conditioned hiperalgesia: Evidence for the conditional compensatory response theory of morphine tolerance. Paper presented at the meeting of the Eastern Psychological Association, Philadelphia, 1979.

3. Siegel. S.. Hinson. R. E., \& Krank, M. D. Pavlovian conditioning and heroin "overdose." Paper presented at the meering of the Psychonomic Society. Phoenix, 1979.

\section{REFERENCES}

Alvarez-Buyli,a, R., \& Dr. Alvarez-Buylla, E. R. Hypoglycemic conditioned reflex in rats: Preliminary study of its mechanisms. Journal of Comparative and Physiological Psychology. 1975, 88, 155-160.

Altanez-Buylla, R., Sequna, E.. \& Di: Alvartz-Buylla, E. R. A study of the afferent path of hypoglycemic reflex of insulin. Acta Physiologica Latinoamericana, 1961, 11, 43-50.

Bondar, R. J. L., \& MEAD, D. Evaluation of glucose-6phosphate dehydrogenase from leuconostoc mesenteroides in the hexokinase method for delermining glucose in serum. Clinical Chemistry' 1974, 20, 586.

Chen, M.. Woods, S. C.. \& Portr, D., Jr. Effect of cerebral intraventricular insulin on pancreatic insulin secretion in the dog. Diaheles, 1975, 24, 910-914.

Drutsci, R. Conditioned hypoglycemia: A mechanism for saccharin-induced sensiticity to insulin in the rat. Journal of Comparative and Physiological Psychology, 1974, 86, 350-358.

Eıкьльом, R., \& Striwakt. J. Conditioned temperature effects using morphine as the unconditioned stimulus. Psuchopharmacology, 1979, 61. 31-38.

Fitzgerald, R. D., Martin, G, K., \& O’Briren, J. H. Influence of vagal activity on classically conditioned heart rate in rats. Journal of Comparative and Physiological Psychology. 1973. 83, 485-491.

LI, J. C. R. Statistical inference (Vol. 1). Ann Arbor, Mich: Edwards, 1964.

SIEGEL, S. Conditioning of insulin-induced glycemia. Journal of Comparative and Physiological Psuchology. 1972, 78, 233-241.

SiEges, S. Conditioning insulin effects. Journal of Comparative and Phvsiological Psychology, 1975. 89. 189-199. (a)

Sitgki, $S$. Evidence from rats that morphine tolerance is a learned response. Journal of Comparative and Phusiological Psychology, 1975, 89, 498-506. (b)

SIEGEL, S. Morphine analgesic tolerance: Its situation specificity supports a Pavlovian conditioning model. Science, 1976, 193. 323-325.

SIEGEL, S. Morphine tolerance acquisition as an associative process. Journal of Experimental Psycholngl: Animal Behavior Processes, 1977, 3, 1-13.

SIEGEL, S. Tolerance to the hyperthermic effect of morphine in the rat is a learned response. Journal of Comparative and Physiological Psychology, 1978, 92. 1137-1149.

Woops, S. C. Conditioned hypoglycemia: Effect of vagotomy and pharmacological blockade. American Journal of Phusiologr. 1972. 223. 1424-1427.

Woods, S. C. Conditioned hypoglycemia. Journal of Comparative and Physiological Pswholoy. 1976, 90, 1164-1168.

Woods, S. C., \& Kul Kosky, P. J. Classically conditioned changes of blood glucose level. PSwhosmatic Medicine. 1976, 38. $201-219$.

Woods, S., Makous, W., \& Hurron, R. A. A new technique for conditioned hypoglycemia. Psvchonomic Science, 1968, 10, 389-390.

Woods, S. C., Makous, W.. \& Hutron, R. A. Temporal parameters of conditioned hypoglycemia. Journal of Comparative and Physiological Psychology, 1969.69. 301-307.

Woons, S. C., \& Porte, D., JR. Neural control of the endocrine pancreas. Physiological Reviews, 1974, 54, 596-619.

Woods, S. C., \& Porte, D., JR. Effect of intracisternal insulin upon plasma glucose and insulin in the dog. Diabetes, 1975 , 24, 905-909.

(Received for publication January 15, 1980; revision accepted March 27, 1980.) 